\title{
Efficient generation of a perfect optical vortex by using a phase optical element
}

\author{
V.V. Kotlyar ${ }^{1,2}$, A.A. Kovalev ${ }^{1,2}$, A.P. Porfirev ${ }^{1,2}$ \\ ${ }^{1}$ Image Processing Systems Institute - Branch of the Federal Scientific Research Centre "Crystallography and Photonics" of Russian Academy of Sciences, 151 \\ Molodogvardeyskaya st., 443001, Samara, Russia \\ ${ }^{2}$ Samara National Research University, 34 Moskovskoe Shosse, 443086, Samara, Russia
}

\begin{abstract}
We consider generation of a perfect optical vortex by three elements: (i) amplitude-phase element with its transmission being proportional to the Bessel function, (ii) optimal element with the transmission proportional to the sign of the Bessel function, and (3) helical axicon. Maximal intensity of light on the ring is shown to be achieved by using the optimal element. Thickness of the light ring generated by the axicon is approximately two times higher than that for other elements. For perfect optical vortices with the radius of several wavelengths we detect the range of topological charges, within which the ring radius is almost independent on them.
\end{abstract}

Keywords: optical vortex; perfect optical vortex; topological charge; axicon; Bessel function; Fourier optics, Fraunhofer diffraction

\section{Introduction}

In [1], a "perfect" optical vortex (POV) has been considered. Its radius is independent on the topological charge. In [1], POVs are generated by using a phase optical element consisting of a set of concentric rings, the thickness of each of which approximates the delta function. In [2], POVs are generated by using a conical axicon and a spiral phase plate (SPP). In both works, the POV is generated approximately and its quality turned out to be low. In [3], a narrow ring is imaged by using a $4 \mathrm{f}$-setup, but this way does not allow generation of a POV in the focus of a high-aperture objective for optical manipulation. In [4], instead of the axicon, an amplitude-phase optical element approximating the Bessel mode is proposed. The element in [4] is closest to the optimal phase filter proposed in our work. Generation of POV is compared by using 1) an amplitude-phase light field described by a Bessel mode of limited radius, 2) by an optimal phase light field, described in [5] and 3) by the phase field generated by using a conical axicon and SPP [2].

\section{Generation of the "perfect" optical vortex by using an amplitude-phase optical element}

"Perfect" optical vortex [1] has the following complex amplitude:

$$
E_{0}(\rho, \theta)=\delta\left(\rho-\rho_{0}\right) \exp (\operatorname{in} \theta) \text {, }
$$

where $\delta(x)$ is the Dirac delta-function, $(\rho, \theta)$ are polar coordinates in the Fourier-plane of a spherical lens, $n$ is the vortex topological charge. It is seen in Eq. (1) that the radius of an infinitely thin ring $\rho_{0}$ does not depend on the topological charge. The POV (1) can be generated by using an ideal Bessel mode in the focal plane of the lens:

$$
F_{0}(r, \varphi)=J_{n}(\alpha r) \exp (\operatorname{in} \varphi)
$$

where dimensional parameter $\alpha=k \rho_{0} / f$ determines the scale of the $n$-th order Bessel function of the first kind $J_{n}(x)$. radius of the ring with maximal intensity is $\rho_{0}=\alpha f / k$, where $k$ is the wavenumber of a monochromatic coherent light, $f$ is the focal length of the lens. For generation of the field (2) and amplitude mask is needed. In addition, the Bessel function in Eq. (2) is in practice bounded by a circular aperture or illuminated by a Gaussian beam. Both these reasons distort the POV and lead to low energy efficiency, weak dependence of the on-ring maximal intensity on the topological charge, and to a wider ring.

\section{Generation of the "perfect" optical vortex by using an optimal phase optical element}

Here under the optimal element we mean such optical element that directs the major part of the light into the ring of a specified radius. Transmission of such element is [5]:

$$
F_{2}(r, \varphi)=\operatorname{circ}\left(\frac{r}{R}\right) \operatorname{sgn} J_{n}(\alpha r) \exp (\operatorname{in} \varphi)
$$


In the focus of the lens, the field from the complex amplitude (3) reads as

$$
E_{2}(\rho, \theta)=(-i)^{n+1}\left(\frac{k}{f}\right) \mathrm{e}^{i n \theta} \sum_{m=0}^{N-1}(-1)^{m} \int_{r_{m}}^{r_{m+1}} J_{n}\left(\frac{k \rho r}{f}\right) r d r
$$

where $r_{0}=0, r_{m}=\gamma_{n, m} / \alpha, m=1,2, \ldots, N, \quad r_{N}=R$. Putting $\rho=\alpha f / k$ in Eq. (4), the argument of the Bessel function becomes independent on the parameters $f$ and $k$ and equals $\alpha r$. It can be shown that at $\rho_{0}=\gamma_{n, N} f /(k R)$ all terms in the sum are positive and their contribution into the light field on the ring is maximal. For the radius of the POV ring to be independent of the topological charge, close roots of the Bessel function need to be chosen: $\gamma_{n, N}=\gamma_{m, M}$.

\section{Generation of a "perfect" optical vortex by using a axicon}

The POV is often generated by using a conical axicon and a SPP [2]. When light passes through such setup, it is equivalent to passing an element with the following transmittance function:

$$
F_{3}(r, \varphi)=\operatorname{circ}\left(\frac{r}{R}\right) \exp (i \alpha r+i n \varphi)
$$

For the first time, optical element (5) was considered in [6] for generation of light pipes. It was also studied in [7-9]. Instead of scaling factor of the Bessel function, here the parameter $\alpha$ determines an axicon parameter, related with the vertex-angle of the generated conical wave. It is supposed in [2] that in the focus of a spherical lens the light field (5) generates a POV with its amplitude distribution described by the function $E_{3}(\rho, \theta) \exp \left[-\left(\rho-\rho_{0}\right)^{2} / \Delta \rho^{2}\right] \exp (\operatorname{in} \theta)$. It is a simplification and the complex amplitude of the POV is defined by a Fourier transform of the function (5), taking a much more complex form [6]. The intensity on the ring is lower than that for the optimal element (3), but higher than for the amplitude element. The intensity on the ring depends on the topological charge, while the ring thickness is approximately two times larger than in previous cases.

\section{Simulation results}

In this section, we describe the simulation results of generating the POV by using the considered above optical elements. The simulation parameters are as follows: wavelength of light $\lambda=532 \mathrm{~nm}$, circular aperture radius is $R=20 \mathrm{~mm}$, and the focal length of an ideal spherical lens $f=100 \mathrm{~mm}$, while the Bessel function's scale factor $\alpha$ is chosen so that $\alpha R=\gamma_{1,20}=63,6114$, where $\gamma_{1,20}$ is 20th zero of the first-order Bessel function $(v=20, n=1)$. The POV was simulated for two topological charges: $n=1$ and $n=14$, while the other parameters were kept unchanged. Note that for the Bessel function of order $n=14$ we chose the 14th root $(v=14)$ because $\gamma_{14 ; 14} \approx \gamma_{1 ; 20}=63.6114$. Figure 1 shows the absolute values of two Bessel functions, $\left|J_{1}\left(\gamma_{1 ; 20} x / R\right)\right|$ and $\left|J_{14}\left(\gamma_{14 ; 14} x / R\right)\right|$. It is seen in Fig. 1 that both functions are seen to take a zero value at $x=R$.
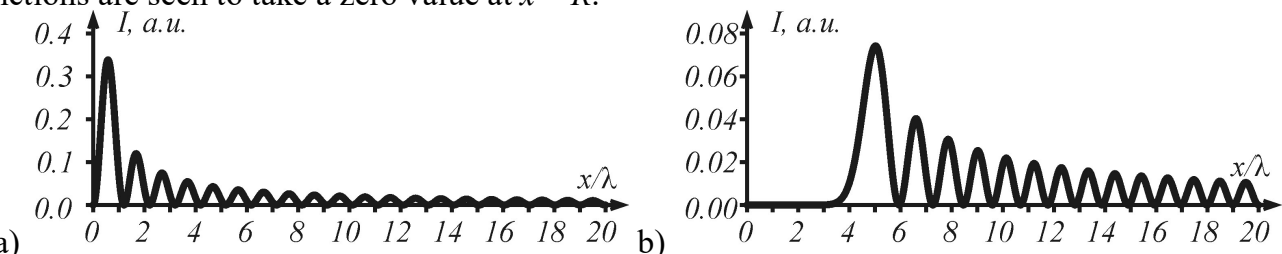

Fig. 1. Absolute values of the Bessel functions $\left|J_{1}\left(\gamma_{1,20} x / R\right)\right|$ (a) and $\left|J_{14}\left(\gamma_{14,14} x / R\right)\right|$ (b), bounded by the radius $R$.

Fig. 2 shows intensity distributions of the POV in the Fourier plane of a spherical lens, obtained with an initial light field in the form of a bounded Bessel mode. Parameters of the calculated POVs are given in Table. 1. It is seen in Table 1 that the POV radius was not changed with changing of the topological charge. At the chosen parameters, the POV radius is $\rho_{0} \approx 50,62 \lambda$. This value is different from the value in Table 1 by only $3 \%$. Maximal intensity of the POV decreased by only $5 \%$ with an increase in the vortex topological charge by almost an order of magnitude. Note that, according to the theory, with the chosen simulation parameters the maximum intensity in Fig. 2 should be equal to $I_{1}\left(\rho_{0}\right)=[k R /(\pi \alpha f)]^{2} \approx 0,015816$. It is consistent with the value in Table 1 . Since the radius of the ring has not changed and the radius of the aperture $R$ of the optical element has not changed either, the width of the ring should not change. Table 1 shows that, indeed, the ring thickness does not change when the topological charge of the optical vortex changes. According to the theory, the ring thickness at the selected simulation parameters should be equal to $\mathrm{FWHM}=5 / 2 \lambda$. This value differs by $11 \%$ from the value of the ring thickness in Table 1 . 


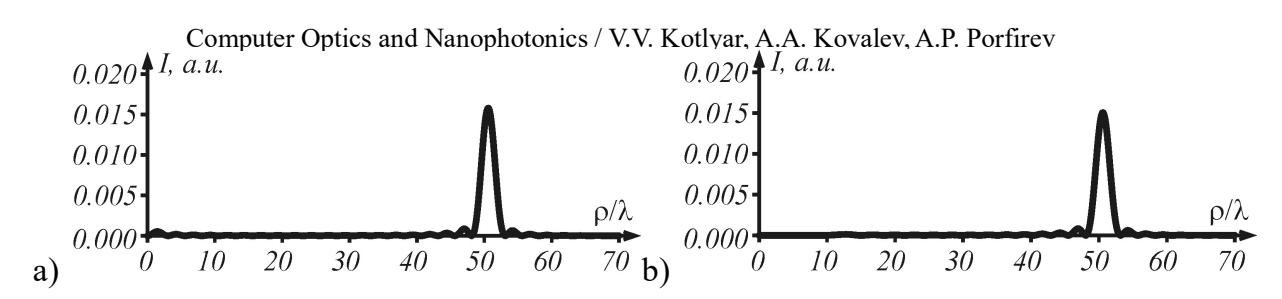

Fig. 2. Intensity distributions of the POV with $n=1$ (a) and $n=14$ (b) for the initial light filed in a form of the Bessel mode.

Table 1. Comparison of the parameters of POV, generated with the initial optical field in Eq. (6) (bounded Bessel function) at different topological charges $n$.

\begin{tabular}{lll} 
Topological charge & $n=1$ & $n=14$ \\
\hline Radius of maximum intensity ring, $\rho_{0}, \lambda$ & 50.781563 & 50.781563 \\
\hline Maximum intensity, $I_{\max }$ (a.u.) & 0.0157968 & 0.0150522 \\
\hline Ring thickness, FWHM, $\lambda$ & 2.244489 & 2.244489
\end{tabular}

Now we consider generation of the POV by using the optimal phase element (3). Fig. 3 shows the intensity distributions for the initial light field (3). Table 2 shows the computed parameters of the POV from Fig. 3. From Table 2, the POV radius became slightly smaller than that in Fig. 2 (less by just $0.3 \%$ ). The radius remained almost unchanged when the topological charge was increased by a factor of 14. The intensity at the ring is almost 100 times greater than the intensity for the POV in Fig. 2. We note that with an increase of the topological charge by a factor of 14 , the intensity on the ring decreased by only $2 \%$. The ring thickness became smaller by approximately $14 \%$ compared to the thickness of the ring in Fig. 2 . The thickness of the ring is preserved when the topological charge of the optical vortex changes. Fig. 3 shows that side lobes have increased.
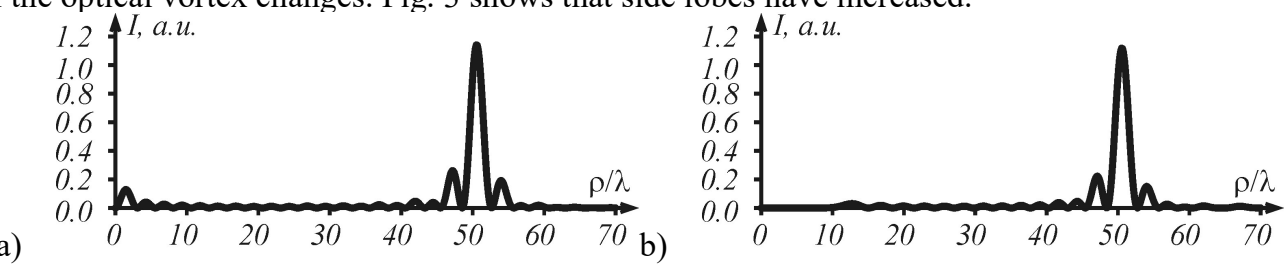

Fig. 3. Intensity distributions of the POV at $n=1$ (a) and $n=14$ (b) for the initial light field (3).

Table 2. Comparison of parameters of the POV, generated with the optimal phase element [Eq. (3)] at different topological charges $n$.

\begin{tabular}{lll} 
Topological charge & $n=1$ & $n=14$ \\
\hline Radius of maximum intensity ring, $\rho_{0}, \lambda$ & 50,641283 & 50,641283 \\
\hline Maximum intensity, $I_{\max }$ (a.u.) & 1,140685 & 1,1181689 \\
\hline Ring thickness, FWHM, $\lambda$ & 1,9639279 & 1,9639279
\end{tabular}

Next, we consider generation of the POV by using a helical axicon (5). Fig. 4 shows intensity distributions for the initial light field (5), while the Table 3 shows the computed parameters of this POV. From Fig. 4 and Table 3, the POV ring thickness is approximately 2.5 times larger than the thickness of the ring in Fig. 2.

In addition, with increasing topological charge of the vortex, the ring thickness increases by a factor of 1.3.

a)
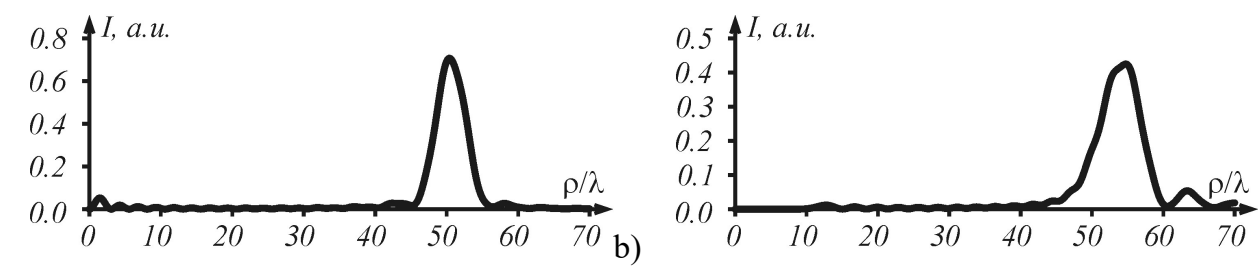

Fig. 4. Intensity distributions of the POV at $n=1$ (a) and $n=14$ (b) for the initial light field (5).

Table 3. Comparison of Parameters of the POV generated by using a spiral axicon [Eq. (25)] at different topological charges $n$.

Topological charge $\quad n=1 \quad n=14$

\begin{tabular}{lll}
\hline Radius of maximum intensity ring, $\rho_{0}, \lambda$ & 50,501002 & 54,849699 \\
\hline Maximum intensity, $I_{\max }$ (a.u.) & 0,7070332 & 0,4249419 \\
\hline Ring thickness, FWHM, $\lambda$ & 4,9098196 & 6,5931864
\end{tabular}


Computer Optics and Nanophotonics / V.V. Kotlyar, A.A. Kovalev, A.P. Porfirev

An increase of the ring thickness (Fig. 4) with increasing $n$ leads to the decreasing intensity on this ring. From Table 3 , it is seen that the maximal intensity on the ROV ring (Fig. 3) decreases 1.7 times as the number $n$ increases by a factor of 14 . And even the radius of the maximal intensity ring increases by $8 \%$. In this case, the thickness of the ring and the maximum intensity almost do not change with increasing topological charge of the optical vortex from 1 to 14 . The only drawback of the POV generated by the element (3) is an increased level of side lobes, which constitute about $20 \%$ of the maximum intensity.

So, the simulation has shown that among the three optical elements for generating the POV, the optimal phase element in Eq. (3) is the best one, since the narrowest ring $(\mathrm{FWHM}=1,96 \lambda=0.39 \lambda f / R)$ is generated in this case with the maximum intensity being 1.6 times higher than that from the spiral axicon in Eq. (5).

Above, the topological charge $n$ and the scaling factor of the axicon $\alpha$ were chosen so that the product $\alpha R$ remained approximately the same and was equal to the root of the Bessel function. Now we consider the case when this is not so. Let this product be arbitrary and let the optimal element or axicon with a diameter of $2 R=40 \lambda$ to generate a POV by using a lens with a focal length $f=100 \lambda$. Fig. 5 shows the dependence of the light ring thickness (at half-maximum of the intensity) on the radius of the ring.

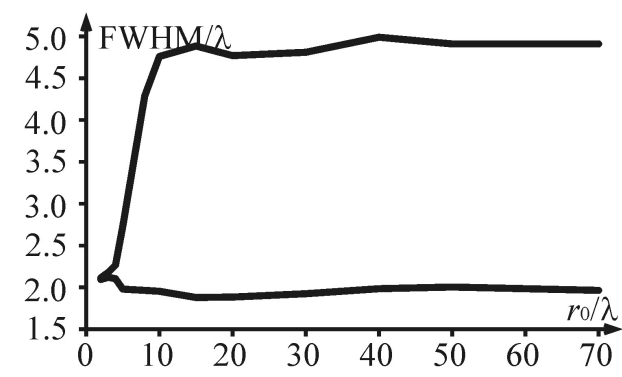

Fig. 5. Thickness of light ring vs. its radius. Upper curve - axicon (5), lower curve - optimal element (3).

For the POV radius $r_{0}=2 \lambda$, both elements work identically. Both of them generate a light ring with a radius of about $r_{0}$ and with about same thickness. At the same time, for the optimal element the maximal energy is approximately $30 \%$ higher. This is explained by the fact that, despite the same width at the level of half-maximum, the thickness at a lower intensity level is larger for the axicon. When $r_{0}=3 \lambda$, both elements form a ring of wrong radius of about $2 \lambda$. However, the maximum energy for the optimal element is $86 \%$ higher. When $r_{0}=4 \lambda$, the optimal element generates a ring of radius $4.1 \lambda$, while the axicon generates two light rings, one of which has a radius $1.9 \lambda$, and the second $-4.7 \lambda$. When $r_{0}=5 \lambda$, both elements generate two light rings with radii $5 \lambda$ and $1.5 \lambda$. Further, for $r_{0}>5 \lambda$, both elements generate a light ring of radius $r_{0}$. With increasing $r_{0}$ up to $r_{0}=70 \lambda$, the ring thickness remains practically unchanged, but in all cases the ring generated by the axicon is about 2 to 2.5 times wider.

Now we consider the dependence of the radius of the generated light ring on the topological charge. Let the optimal element (3) or the axicon (5) of the diameter $2 R$ to generate a POV by using a lens with a focal length $f=100 \lambda$. Fig. 6 shows the dependence of the radius of the generated light ring on the topological charge for elements of radius $R=10 \lambda$ (Fig. 6a), $R=20 \lambda$ (Fig. 6b), and $R=$ $30 \lambda$ (Fig. 6c).

a)
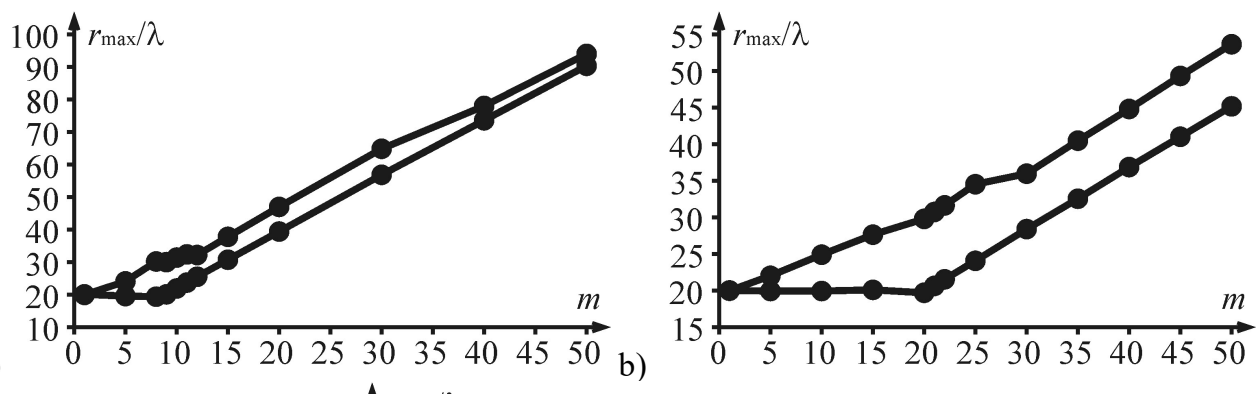

c)

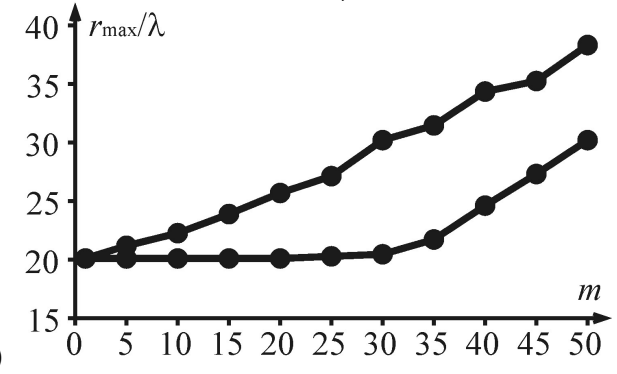

Fig. 6. Radius of the generated light ring vs. the topological charge for different element radii: $R=10 \lambda(a), R=20 \lambda(b)$, and $R=30 \lambda(c)$. Upper curve - axicon $(5)$, lower curve - optimal element (3) 
Computer Optics and Nanophotonics / V.V. Kotlyar, A.A. Kovalev, A.P. Porfirev

It is seen in Fig. 6 that with using the axicon (5) the light ring radius rises with the topological charge nearly linearly. When using the optimal element (3), the radius is almost constant for the topological charges up to $R / \lambda$. With larger topological charges, the radius begins to increase linearly at about the same rate as for the axicon.

\section{Experiment}

For experimental study of the optical elements for generating the POVs we used an optical setup shown in Fig. 7. The fundamental Gaussian beam was a light source generated by a solid-state laser $L(\lambda=532 \mathrm{~nm})$. The laser beam was expanded and collimated using a system composed of a 40- $\mu \mathrm{m}$ pinhole $P H$ and a lens $L_{1}\left(f_{1}=250 \mathrm{~mm}\right)$. Then the beam illuminated the display of a spatial light modulator SLM (PLUTO VIS, $1920 \times 1080$ resolution, with $8 \mu \mathrm{m}$ pixels). The diaphragm $D_{1}$ was used to separate the central bright spot from the surrounding dark and bright rings caused by diffraction by the pinhole. Further, using the lenses $L_{2}\left(f_{2}=\right.$ $350 \mathrm{~mm})$ and $L_{3}\left(f_{3}=150 \mathrm{~mm}\right)$ and the diaphragm $D_{2}$, spatial filtering of the phase-modulated laser beam reflected at the SLM display was performed. Using a lens $L_{4}\left(f_{4}=500 \mathrm{~mm}\right)$, the laser beam was focused on the CCD array of a video camera LOMO TC 1000 (pixel size $1.67 \times 1.67 \mu \mathrm{m}$ ). To generate the POVs, we used the phase masks shown in Fig. 8, which were output to the SLM display. To separate the non-modulated beam reflected at the display and the phase-modulated beam, a linear phase mask was superimposed on the initial phase mask.

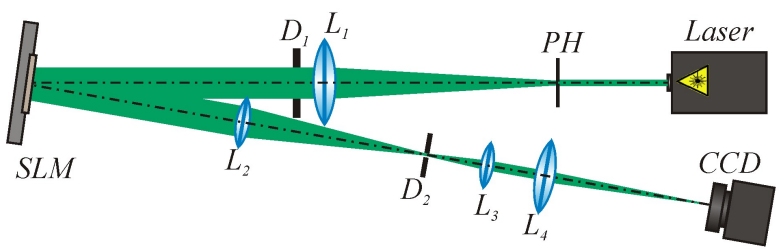

Fig. 7. Experimental setup: $L$ is a solid-state laser $(\lambda=532 \mathrm{~nm}) ; P H$ is a $40-\mu \mathrm{m}$ pinhole; $L_{1}, L_{2}, L_{3}$, and $L_{4}$ are lenses with focal lengths $f_{1}=250 \mathrm{~mm}, f_{2}=350 \mathrm{~mm}, f_{3}=$ $150 \mathrm{~mm}$, and $f_{4}=500 \mathrm{~mm} ; D_{1}$ and $D_{2}$ are diaphragms; SLM is a spatial light modulator PLUTO VIS; and CCD is a video camera LOMO TC-1000.

a)

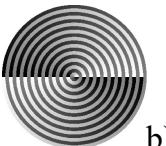

b)

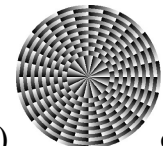

c)

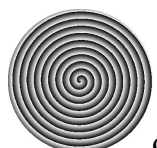

d)

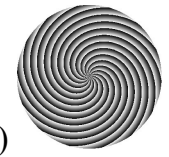

Fig. 6. Phase masks of optical elements to generate a POV with a topological charge (a, c) $n=1$ and (b, d) $n=14$. (a) and (b) depict optimal phase elements and (c) and (d) are for spiral axicons.

Fig. 7 shows the intensity distributions in the focus of lens $L_{4}$ generated by using phase masks for the optimal phase elements with topological charges $n=1$ and $n=14$. The values of the parameters of the resulting POV are given in Table 4.

Figure 8 depicts the intensity distributions in the focus of lens $L_{4}$ generated using phase masks corresponding to the spiral axicons with $n=1$ and $n=14$. The values of the parameters of the resulting POV are given in Table 5. Analyzing the experimentally measured parameters of the POV, we can conclude that their relative values are in good agreement with the simulation results.
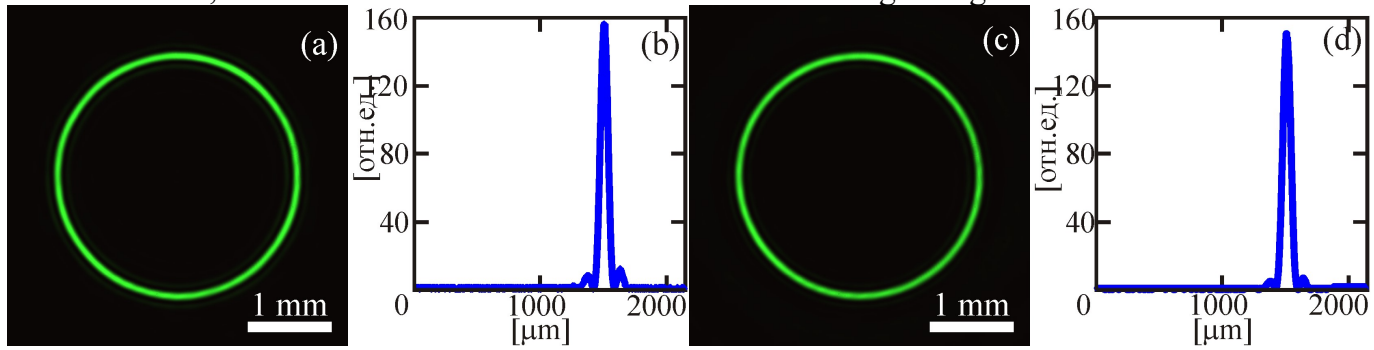

Fig. 9. Intensity distributions of the POV $(a, c)$ and respective profiles depicted from the center to the edge (b, d) obtained by using an optimal phase element with (a, b) $n=1$ and (c, d) $n=14$.

Table 4. Comparison of parameters of POV obtained by using an optimal phase element with topological charges $n=1$ and $n=14$

\begin{tabular}{|l|l|l|}
\hline Topological charge & $n=1$ & $n=14$ \\
\hline Radius of maximum intensity ring, $\mu \mathrm{m}$ & $1491.0 \pm 2.0$ & $1496.5 \pm 2.0$ \\
\hline Maximum intensity, a.u. & $156.0 \pm 0.5$ & $151.0 \pm 0.5$ \\
\hline Ring thickness, FWHM, $\mu \mathrm{m}$ & $70.0 \pm 2.0$ & $73.0 \pm 2.0$ \\
\hline
\end{tabular}




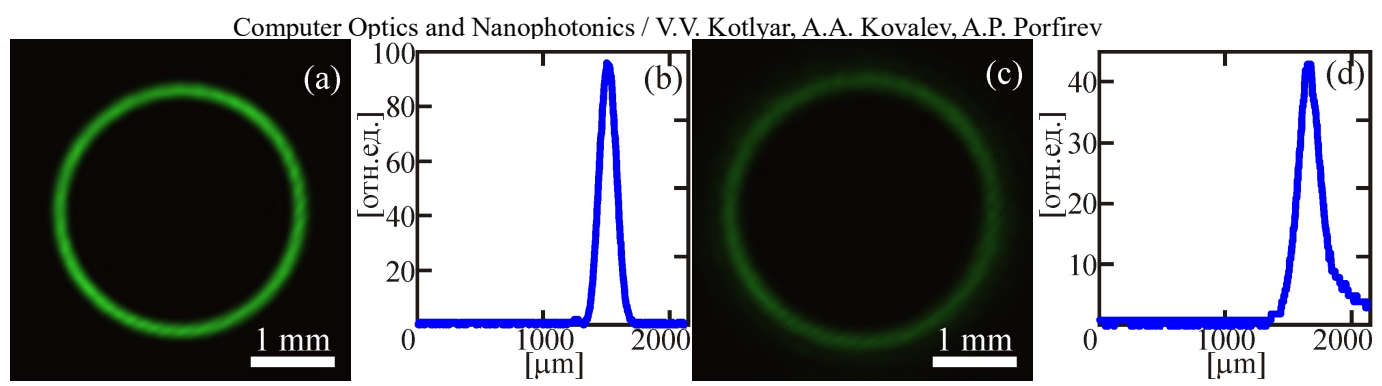

Fig. 10. Intensity pattern of a POV (a, c), with the respective profiles depicted from the center to the edge (right $\mathrm{b}, \mathrm{d})$ for a spiral axicon with (a,b) $n=1$ and (c,d) $n=$ 14.

Table 5. Comparison of parameters of POV obtained by using a spiral axicon with topological charges $n=1$ and $n=14$.

\begin{tabular}{|l|l|l|}
\hline Topological charge & $n=1$ & $n=14$ \\
\hline Radius of maximum intensity ring, $\mu \mathrm{m}$ & $1498.0 \pm 2.0$ & $1655.0 \pm 2.0$ \\
\hline Maximum intensity, a.u. & $96.0 \pm 0.5$ & $43.0 \pm 0.5$ \\
\hline Ring thickness, FWHM, $\mu \mathrm{m}$ & $158.0 \pm 2.0$ & $206.0 \pm 2.0$ \\
\hline
\end{tabular}

\section{Conclusion}

In this work, generation of a perfect optical vortex by three different optical elements is considered: amplitude-phase element with a transmission proportional to the Bessel mode, an optimal phase element and a vortex axicon. It is shown that using any of these three optical elements leads to generation of light rings with the same radius, which depends little on the topological charge of the optical vortex. However, if the POV radius is equal to several wavelengths, then it depends on the topological charge. For the axicon, this dependence is almost linear, while for the optimal element this dependence is almost absent for topological charges that are smaller than the ratio of the element's radius to the wavelength. The intensity of light on the ring is greater (with other conditions being equal) for the optimal phase element. The intensity of all three rings depends little on the topological charge. The thickness of the light ring generated by the vortex axicon is approximately twice larger compared to that of the other two rings. Thus, the optimal filter (3), studied for the first time in [3], is the best candidate for generation of a perfect optical vortex.

\section{Acknowledgements}

The work was funded by the Russian Science Foundation (RSF) grant \# 17-19-01186 (the results pertaining to the experimental generation of perfect optical vortices presented in Section 6), as well as by the Russian Foundation for Basic Research (RFBR) grant \# 17-47-630420 (the results pertaining to the numerical simulation of the perfect optical vortices generated by different elements presented in Sections 1-5).

\section{References}

[1] Ostrovsky AS, Rickenstorff-Parrao C, Arrizon V. Generation of the "perfect" optical vortex using a liquid-crystal spatial light modulator. Optics Letters 2013; 38(4): 534-536.

[2] Chen M, Mazilu M, Arita Y, Wright EM, Dholakia K. Dynamics of microparticles trapped in a perfect vortex beam. Optics Letters 2013; 38(22): 4919-4922.

[3] Li P, Zhang Y, Liu S, Ma C, Han L, Cheng H, Zhao J. Generation of perfect vectorial vortex beams. Opt. lett. 2016; 41(10): 2205-2208.

[4] García-García J, Rickenstorff-Parrao C, Ramos-García R, Arrizón V, Ostrovsky A. Simple technique for generating the perfect optical vortex. Optics Letters 2014; 39(18): 5305-5308.

[5] Fedotowsky A, Lehovec K. Optimal filter design for annular imaging. Applied Optics 1974; 13(12): $2919-2923$.

[6] Kotlyar VV, Kovalev AA, Skidanov RV, Moiseev OY, Soifer VA. Diffraction of a finite-radius plane wave and a Gaussian beam by a helical axicon and a spiral phase plate. Journal of the Optical Society of America A. 2007; 24(7): 1955-1964.

[7] Kotlyar VV, Kovalev AA, Cojoc D, Garbin V, Ferrari E. Diffraction of a Gaussian beam by a spiral axicon. Computer Optics 2006; 30 : 30-35.

[8] Kotlya, VV, Kovalev AA, Soifer VA, Davis JA, Tuvey CS, Cottrell DM. Diffraction of a finite-radius plane wave by a spiral axicon and by a spiral phase plate: comparison. Computer Optics 2006; 30: 36-43.

[9] Degtyarev SA, Khonina SN, Podlipnov VV. Formation of spiral intensity by binary vortical axicon. Computer Optics 2014; $38(2): 237--242$. 